\title{
Observing the high redshift Universe with Euclid
}

\author{
René Laureijs ${ }^{1}$ on behalf of the Euclid Collaboration
}

\author{
${ }^{1}$ European Space Agency, ESTEC, Keplerlaan 1 \\ PO Box 299, NL-2200 AG, Noordwijk, the Netherlands \\ email: rene.laureijs@esa.int
}

\begin{abstract}
Euclid enables the exploration of large sky areas with diffraction limited resolution in the optical and near-infrared, and is sensitive enough to detect targets at cosmological distances. This combination of capabilities gives Euclid a clear advantage over telescope facilities with larger apertures, both on ground and in space. The decision to mount in the NISP instrument one extra grism for the wavelength range 0.92-1.3 $\mu \mathrm{m}$ with a spectral resolution of $\mathrm{R} \approx 260$ makes possible a rest-frame UV survey of the early Universe in the redshift range $6.5<z<9.7$. Euclid's standard imaging with VIS in the $0.55-0.9 \mu \mathrm{m}$ band and with NISP in the Y, J, H bands provide complementary photometry for further target identification and characterization. Euclid is a suitable facility to discover and map the spatial distribution of rare high-redshift targets and to collect statistically relevant samples, in particular of high redshift Ly $\alpha$ emitters and QSOs, which can be used as signposts of the cosmic structures. The Euclid surveys are also a starting point for deeper follow up observations of the individual high- $z$ objects. We present the Euclid mission and discuss the detectability of high- $z$ objects to probe the epoch of ionization.
\end{abstract}

Keywords. Euclid, space vehicles: instruments, early universe

\section{Introduction}

Even though the Euclid mission of the European Space Agency has been designed for the measurement of two dark energy probes - Weak Lensing and Galaxy Clustering at $z<2$, it also enables studies of the high redshift Universe (Laureijs et al., 2011). During the design phase of the mission a further optimisation was implemented for the Galaxy Clustering probe to maximize its signal by reducing the spectroscopic redshift range. The resulting mechanical modification left room in the near-infrared instrument to include one additional grism in the short wavelength range $0.92<\lambda<1.3 \mu \mathrm{m}$ (Costille et al., 2016), providing a spectroscopic channel which is extremely suitable for observing the high redshift Universe.

The Euclid mission is required to survey large areas of the sky in order to achieve its required Dark Energy figure of merit. Its wide survey covers 15,000 $\mathrm{deg}^{2}$. In addition, the deep survey covers $40 \mathrm{deg}^{2}$ and will be $2 \mathrm{mag}$ deeper than the wide survey. The deep survey is required for calibrations and is also reserved for additional scientific topics.

Recognizing the opportunity, the Euclid Science Team unanimously decided that the "extra" short wavelength grism of the near-infrared slitless spectrometer shall be used for the deep survey. The observations shall be optimised for the investigation of the high redshift Universe. This channel is well suited to detect very high redshift lines beyond $z>2$. In Section 2 we will describe in more detail the Euclid mission and its status, the Euclid surveys, and the science capabilities, in the light of the spectroscopy with the short wavelength channel. Section 3 gives an overview of the high redshift Universe science which can be addressed. Our conclusions are given in Section 4 . 
Table 1. Observational parameters for the Euclid deep survey.

\begin{tabular}{|c|c|c|c|c|}
\hline Instrument & Channel & $\begin{array}{l}\text { Range(s) } \\
{[\mu \mathbf{m}]}\end{array}$ & $\begin{array}{l}\text { Detection } \\
\text { limit }\end{array}$ & Comment \\
\hline $\begin{array}{l}\text { NISP-S } \\
\text { NISP-L } \\
\text { NISP-P } \\
\text { VIS }\end{array}$ & $\begin{array}{l}\text { Short Wavelength Grism } \\
\text { Long Wavelength Grism } \\
\text { NIR photometry } \\
\text { visual imaging }\end{array}$ & $\begin{array}{l}(0.92-1.30) \\
(1.25-1.85) \\
Y(0.97-1.19) \\
J(1.19-1.54) \\
\mathrm{H}(1.54-2.00) \\
0.55-0.90\end{array}$ & $\begin{array}{l}6 \times 10^{-17} \mathrm{erg} \mathrm{cm}^{-2} \mathrm{~s}^{-1} \\
5 \times 10^{-17} \mathrm{erg} \mathrm{cm}^{-2} \mathrm{~s}^{-1} \\
26.0 \mathrm{mag}(\mathrm{AB}) \\
26.5 \mathrm{mag}(\mathrm{AB})\end{array}$ & $\begin{array}{l}3 \sigma \\
3 \sigma \\
5 \quad \sigma \text { point source } \\
10 \sigma \text { extended source }\end{array}$ \\
\hline
\end{tabular}

This contribution is based on a the NISP "blue grism" Science Definition Report for Euclid, in preparation by the Euclid Collaboration (Brinchmann et al., 2018).

\section{The Euclid Mission}

The Euclid satellite is planned for launch from ESA's spaceport in Kourou in 2021. A Soyuz-Fregat combination will inject the satellite in a wide orbit around the second Sun-Earth Lagrange point at a distance of 1.5 million $\mathrm{km}$ from the Earth. Its nominal mission duration is 6 years and is defined by the time necessary to complete the wide and deep surveys (Racca et al., 2016).

The satellite carries a wide field telescope with a primary mirror of $1.2 \mathrm{~m}$ diameter. By means of a dichroic plate, the light of the telescope is diverted to a visual imager (VIS) and a near infrared spectrometer and imaging photometer (NISP). The two instruments view the same sky area of $0.54 \mathrm{deg}^{2}$ and can measure simultaneously. The VIS instrument performs single-band imaging in the wavelength range $0.55-0.90 \mu \mathrm{m}$ using CCDs. The FWHM of the point spread function in the VIS images will be better than 0.17 arcsec at $800 \mathrm{~nm}$. NISP is capable of two slitless spectroscopy modes and of photometric imaging in three bands using $\mathrm{HgCdTe}$ detectors, see Table 1.

Whilst the wide survey covers $15,000 \mathrm{deg}^{2}$ centred on the galactic poles, the Euclid deep survey covers a total area of $40 \mathrm{deg}^{2}$ and is distributed over three deep fields: (1) EDF-N: $10 \mathrm{deg}^{2}$ circle centred on the north ecliptic pole, (2) EDF-S: $20 \mathrm{deg}^{2}$ circle close to the south ecliptic pole, and (3) EDF-Fornax: $10 \mathrm{deg}^{2}$ circle centred on the Chandra Deep Field South. The final centre positions of the fields are still discussed as these depend on the spacecraft pointing capabilities, sky background, calibration requirements and optimal alignment with existing other surveys. The ecliptic poles have been selected because of their all-year accessibility within the pointing constraints of the satellite.

Like the Euclid wide survey, each deep survey satellite pointing consists of 4 dithers, each is composed of a simultaneous VIS imaging and NISP spectroscopic measurement, followed by photometric exposures in the $\mathrm{Y}, \mathrm{J}$, and $\mathrm{H}$ bands. Unlike the wide survey, a measurement with the short wavelength grism is included, either by replacing a long wavelength grism measurement in one of the dithers, or by a separate additional measurement. Any position of the deep survey is visited at least 40 times to achieve a nominal depth which is 2 mag deeper than the wide survey depth. The pointings are scheduled at different epochs throughout the nominal mission. Spreading the observations in time minimizes source confusion because of the rotation of the position angle of a given source spectrum on the sky. 

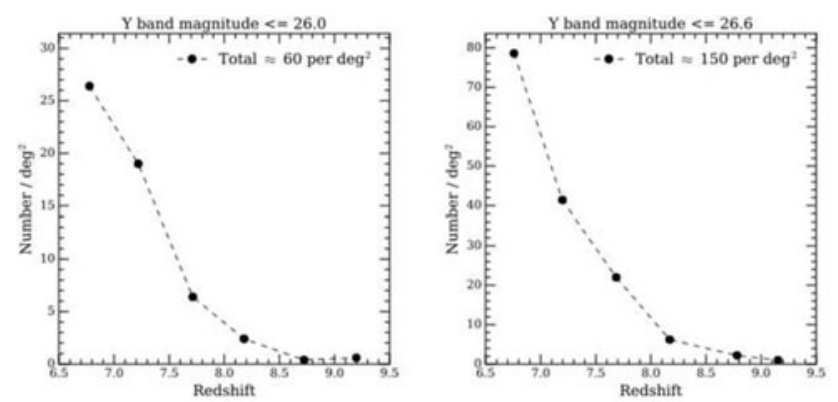

Figure 1. Predicted Ly $\alpha$ number counts. The left-hand panel shows the predicted Ly $\alpha$ number counts per $\operatorname{deg}^{2}$ with $F\left(L y_{\alpha}\right)>6 \times 10^{-17} \mathrm{erg} \mathrm{s}^{-1} \mathrm{~cm}^{-2}$ and with a $>5 \sigma$ detection in the Euclid $\mathrm{Y}$ band $(\mathrm{Y} \leqslant 26.0)$. The right-hand panel shows the same for all galaxies with a $>3 \sigma$ detection $(Y \leqslant 26.6)$

\section{Observing the high redshift Universe}

\subsection{A facility for finding new targets}

The greatest strength of Euclid is its capability to homogeneously cover large areas of sky. The Euclid near-infrared measurements are not compromised by atmospheric emission and benefit from large efficiency gains over similar ground based observations. The Euclid surveys promise to find rare and distant objects: bright galaxies and QSOs, fainter but lensed objects, and objects in a short-lived transient phase. Euclid has the capability to collect useful samples of these rare targets: for statistically relevant studies and for the selection of targets to carry out follow-up observations with facilities which can go deeper than Euclid.

\subsection{Expectations for Ly- $\alpha$ emitters and $Q S O s$}

The spectral window of the short wavelength grism covers the Lyman break in the range $z=6.5-9.7$. This spans the end of the re-ionisation period when the Universe was less than a billion years old, offering the prospect of detecting the first generation of stars and the earliest evolutionary stages of bright galaxies. Euclid's deep field imaging with VIS in the $0.55-0.9 \mu \mathrm{m}$ band and with NISP in the Y, J, H bands provides the complementary photometry for further target identification and characterisation.

Ly- $\alpha$ emission is expected to become increasingly more difficult to detect at $z>6.5$, which is assumed to mark the end of the re-ionization process happening at the higher redshifts. However, bright and very rare Ly- $\alpha$ emitters have been observed suggesting a patchy and inhomogeneous process (e.g. Bagley et al., 2017).

To estimate the number of detectable Ly $\alpha$ emitters in the Euclid deep survey, we performed dedicated simulations using the simulation tools developed for Euclid. Based on the evolution properties at $z<4$ we generated galaxy mock catalogues for the higher redshifts at $6<z<9$. Mock spectroscopy observations were obtained by including a dust attenuation prescription and a constant Ly $\alpha$ escape fraction of 30\% (Brinchmann et al., 2018). In order to be able to extract a spectrum from a slitless grism observation, the position of the target has to be known. The galaxy position can be obtained from the Euclid near-infrared imaging photometry. These Euclid observations are important as there will be no continuum emission from the sources at the shorter wavelengths $(\lambda<0.9$ $\mu \mathrm{m})$. By using the $\mathrm{Y}$ band source detections down to $\mathrm{Y}<26.0 \mathrm{mag}(5 \sigma)$ a survey density of $60 \mathrm{deg}^{-2} \mathrm{Ly}-\alpha$ detections is predicted, see Fig. 1. According to this prescription, about $30 \%$ of the Lyman break population can be detected by Euclid. Conversely, Marchetti et al. (2017) estimated the number detectable Ly- $\alpha$ emitters at $z>6.6$ to be of order only 
one $\mathrm{deg}^{-2}$, or 34 to 73 for the entire Euclid deep survey of $40 \mathrm{deg}^{2}$. It is assumed that these objects have been lensed. These contradictory estimates clearly indicate that the actual deep field observations will impose strong constraints on the model assumptions. Marchetti et al. (2017) mention that SKA will enable the identification of the interloper population of $\mathrm{H} \alpha$ emitters, improving the purity of the sample of Ly- $\alpha$ emitters.

Spectra of both Type 1 and Type 2 AGNs are expected to be rich in strong highionization emission lines in the UV. Our own calculations suggest that we can expect about 140 QSOs with $6<z<8$ for the deep survey. Waters et al. (2016) predict 180 QSOs at $z>8$, if we assume that the WFIRST detection limit is comparable to that of the Euclid deep survey. The numbers are indicative for the level of uncertainty in our estimates. The Euclid deep survey can place strong constraints on the currently unknown high-z AGN evolution, using spectroscopic identification of the emission lines of the objects. These observations will address the formation of supermassive black holes and the physical connection between galaxies and the black holes in their centres; two key questions in astrophysics.

The discovery of an extremely massive and dusty star-forming galaxy at $z=6.90$ from submm observations with SPT and ALMA (Strandet et al., 2017) has shown that high amounts of obscuring dust is already formed as early as 800 Myrs after the Big Bang, imposing stronger contraints on the detectability of these targets in the rest frame UV survey with Euclid. This illustrates that the epoch of re-ionisation will likely reveal rare objects with extreme physical properties.

\section{Prospects and Conclusions}

The combination of sky area, wavelength coverage and depth of the Euclid deep survey will produce a unique dataset for the investigation of the high redshift Universe. The galaxy density in the deep survey will be more than about $300,000 \mathrm{deg}^{-2}$, and only a very tiny fraction, less than $10^{-4}$, of the objects are at redshifts $z>6$. The Euclid collaboration is presently developing dedicated processing algorithms, and careful analysis is required to extract the high redshift sources. It will be a challenge to reliably extract these targets from the Euclid data also in view of the massive volume of pixel data. Once the higher data products are available, we have the opportunity to observe cosmic structures during the period of active growth and assembly. The object detection rates can be used to firmly constrain reionisation models and early galaxy evolution scenarios.

The first Euclid deep survey related data will be made public before the middle of the next decade. New high performance facilities will then be operational for follow up: JWST, SKA, Subaru PFS, and possibly at a later stage ELT and WFIRST.

\section{References}

Bagley, M. B., Scarlata, C., Henry, A., et al. 2017, ApJ, 837, 11

Brinchmann, J. et al. 2018, NISP Blue Grism Science Definition Report, in preparation Costille, A., Caillat, A., Rossin, C., et al. 2016, Proc. of SPIE, 9912, 99122C

Laureijs, R., Amiaux, J., Arduini, S. et al. 2011, ArXiv, 1110.3193

Marchetti, L. Serjeant S., Vaccari M. 2017, MNRAS, 470, 5007

Racca, G. D., Laureijs, R., Stagnaro, L. et al. 2016, Proc. of SPIE, 9904, 990400

Strandet M., L., Weiss, A., De Breuck, C. et al. 2017, ApJ, 842, L15

Waters, D., Di Matteo, T., Feng, Y., Wilkins, S. M., Croft, R. A. C., et al. 2017, ApJ, 842, L15 